\title{
Neutral MSSM Higgs-boson production with heavy quarks: NLO supersymmetric QCD corrections
}

\author{
S. Dittmaier, ${ }^{1}$ P. Häfliger, ${ }^{2,3}$ M. Krämer, ${ }^{4}$ M. Spira, ${ }^{3}$ and M. Walser ${ }^{3,5}$ \\ ${ }^{1}$ Physikalisches Institut, Albert-Ludwigs-Universität Freiburg, D-79104 Freiburg, Germany \\ ${ }^{2}$ Institute for Particle Physics, ETH Zürich, CH-8093 Zürich, Switzerland \\ ${ }^{3}$ Paul Scherrer Institut, CH-5232 Villigen PSI, Switzerland \\ ${ }^{4}$ Institute for Theoretical Particle Physics and Cosmology, RWTH Aachen University, \\ D-52056 Aachen, Germany \\ ${ }^{5}$ Institute for Theoretical Physics, ETH Zürich, CH-8093 Zürich, Switzerland
}

(Received 26 June 2014; published 12 August 2014)

\begin{abstract}
Within the minimal supersymmetric extension of the Standard Model (MSSM) the associated production of neutral Higgs bosons with top and bottom quarks belongs to the most important Higgs-boson production processes at the LHC. At large values of $\operatorname{tg} \beta$, in particular, bottom-Higgs associated production constitutes the dominant production channel within the MSSM. We have calculated the next-to-leading-order supersymmetric QCD corrections to neutral Higgs production through the parton processes $q \bar{q}, g g \rightarrow$ $t \bar{t} / b \bar{b}+h / H / A$ and present results for the total cross sections. The genuine SUSY-QCD corrections are of moderate size for small $\operatorname{tg} \beta$, but can be sizable for large $\operatorname{tg} \beta$. In the latter case the bulk of these corrections can be absorbed into effective bottom Yukawa couplings.
\end{abstract}

DOI: 10.1103/PhysRevD.90.035010

PACS numbers: 14.80.Da, 12.38.Bx

\section{INTRODUCTION}

The Higgs mechanism [1] is a cornerstone of the Standard Model (SM) and its supersymmetric extensions. Even after the discovery [2] of a Higgs boson by the LHC experiments ATLAS and CMS in 2012, Higgs-boson searches belong to the major endeavors at present and future colliders, but now with a particular focus on extended Higgs sectors. The minimal supersymmetric extension of the SM (MSSM) is among the best motivated models providing more than one Higgs boson. In the MSSM two isospin Higgs doublets are introduced in order to generate masses for upand down-type fermions [3]. After electroweak symmetry breaking 3 of the 8 degrees of freedom are absorbed by the $Z$ and $W$ gauge bosons, leaving five states as elementary Higgs particles. These consist of two $C P$-even neutral (scalar) particles $h, H$; one $C P$-odd neutral (pseudoscalar) particle $A$; and two charged bosons $H^{ \pm}$. At leading order the MSSM Higgs sector is fixed by two independent input parameters which are usually chosen as the pseudoscalar Higgs mass $M_{A}$ and $\operatorname{tg} \beta=v_{2} / v_{1}$, the ratio of the two vacuum expectation values. Including the one-loop and dominant two-loop corrections the upper bound of the light scalar Higgs mass is $M_{h} \lesssim 135 \mathrm{GeV}$ [4] for supersymmetric mass scales up to about a TeV. Three-loop results [5] and the resummation of logarithmic corrections from the scalar top sector [6] confirm this upper bound within $1 \mathrm{GeV}$; see the recent numerical analysis [7]. An important property of the bottom Yukawa couplings is their enhancement for large values of $\operatorname{tg} \beta$. The top Yukawa couplings, on the other hand, are suppressed for large $\operatorname{tg} \beta$ [8], unless the light (heavy) scalar Higgs mass is close to its upper (lower) bound, where their couplings become SM-like. The couplings of the various neutral MSSM Higgs bosons to fermions and gauge bosons, normalized to the SM Higgs couplings, are listed in Table I, where the angle $\alpha$ denotes the mixing angle of the scalar Higgs bosons $h, H$. The negative direct searches for neutral MSSM Higgs bosons at LEP2 have led to lower bounds of $M_{h, H}>92.8 \mathrm{GeV}$ and $M_{A}>93.4 \mathrm{GeV}$ [9]. The LEP2 results also exclude the range $0.7<\operatorname{tg} \beta<$ 2.0 in the MSSM, assuming a SUSY scale $M_{\text {SUSY }}=$ $1 \mathrm{TeV}$ [9]. MSSM Higgs-boson searches have continued at the $p \bar{p}$ collider Tevatron (see e.g. [10]), and form the central part of the current and future physics program of the LHC [11]. The proton-proton collider LHC has taken data at the center-of-mass (CM) energies of 7 and $8 \mathrm{TeV}$, and will start operating near its design energy of 13$14 \mathrm{TeV}$ in 2015. The present LHC searches at 7 and $8 \mathrm{TeV}$ $\mathrm{CM}$ energy have excluded parts of the MSSM parameter space for large values of $\operatorname{tg} \beta$ [12]. However, the recent discovery of a resonance with a mass near $125 \mathrm{GeV}$ [2] is a clear indication for the existence of a SM or beyond-theSM Higgs boson. While the properties of the new particle, as determined so far, are consistent with those predicted

TABLE I. MSSM Higgs couplings to $u$ - and $d$-type fermions and gauge bosons $[V=W, Z]$ relative to the SM couplings.

\begin{tabular}{lcccc}
\hline \hline \multicolumn{1}{c}{$\phi$} & & $g_{u}^{\phi}$ & $g_{d}^{\phi}$ & $g_{V}^{\phi}$ \\
\hline SM & $H$ & 1 & 1 & 1 \\
MSSM & $h$ & $\cos \alpha / \sin \beta$ & $-\sin \alpha / \cos \beta$ & $\sin (\beta-\alpha)$ \\
& $H$ & $\sin \alpha / \sin \beta$ & $\cos \alpha / \cos \beta$ & $\cos (\beta-\alpha)$ \\
& $A$ & $1 / \operatorname{tg} \beta$ & $\operatorname{tg} \beta$ & 0 \\
\hline \hline
\end{tabular}


within the SM, the bosonic state at $125 \mathrm{GeV}$ can also be interpreted as a supersymmetric Higgs boson.

Neutral MSSM Higgs boson production at the LHC is dominated by gluon fusion, $g g \rightarrow h / H / A$, and by the associated production of a Higgs boson with bottom quarks. Gluon fusion is most significant at small and moderate $\operatorname{tg} \beta$. At large values of $\operatorname{tg} \beta$, however, bottomHiggs associated production becomes dominant due to the strongly enhanced bottom Yukawa couplings [13]. Higgsboson radiation off top quarks [14] [see Fig. 1(a)]

$$
q \bar{q} / g g \rightarrow t \bar{t}+h / H / A
$$

plays a significant role at the LHC for the light scalar Higgs particle only. The next-to-leading-order (NLO) QCD corrections are identical to those for the SM Higgs boson with modified top and bottom Yukawa couplings, and are thus of moderate size $[15,16]$. The SUSY-QCD corrections, which have been computed in Refs. [17-19], are of moderate size as well. First steps towards more realistic descriptions of these processes have been made in Ref. [20], where top-quark and Higgs-boson decays were modeled in LO precision and QCD parton-shower corrections were matched to the NLO-corrected prediction of the production process. The successful experimental study of $t \bar{t}+h / H / A$ with $h / H / A \rightarrow b \bar{b}$ decays critically depends on the proper control of the huge irreducible background from direct $t \bar{t} b \bar{b}$ and $t \bar{t}+2$ jets production. The NLO QCD corrections to these background processes have been calculated in recent years [21], but further improvements are still necessary to fully exploit LHC data, such as e.g. the inclusion of QCD corrections to the top-quark decays. Progress in this direction is ongoing (see, e.g. Refs. [22-24]).

For large values of $\operatorname{tg} \beta$ Higgs-boson radiation off bottom quarks [14] [see Fig. 1(a)]

$$
q \bar{q} / g g \rightarrow b \bar{b}+h / H / A
$$

constitutes the dominant Higgs-boson production process. The NLO QCD corrections can be inferred from the analogous calculation involving top quarks $[15,16]$. However, they turn out to be numerically enhanced [25]. The main reason is that the integration over small transverse momenta of the final-state bottom quarks generates sizable logarithmic contributions. Those logarithms can be resummed by introducing bottom-quark densities in the proton [26] and by applying the standard Dokshitzer-Gribov-Lipatov-Altarelli-Parisi evolution [27]. In this so-called five-flavor scheme (5FS) the leading-order process is [28]

$$
b \bar{b} \rightarrow h / H / A,
$$

where the transverse momenta of the incoming bottom quarks, their masses, and their off-shellness are neglected at LO. The NLO [29] and NNLO [30] QCD corrections as well as the SUSY-electroweak corrections [31] to these bottom-initiated processes have been calculated. They are of moderate size, if the running bottom Yukawa coupling is introduced at the scale of the corresponding Higgs-boson mass. The fully exclusive $g g \rightarrow b \bar{b}+h / H / A$ process, calculated with four active parton flavors in a four-flavor scheme (4FS), and the 5FS calculation converge at higher perturbative orders, and there is fair numerical agreement between the NLO 4FS and NNLO 5FS cross section predictions [22,23,25,32]. In Ref. [33] a scheme has been proposed to match the 4FS and 5FS cross sections ("Santander matching"). The scheme is based on the observation that the 4FS and 5FS calculations of the cross section are better motivated in the asymptotic limits of small and large Higgs masses, respectively, and combines the two approaches in such a way that they are given variable weight, depending on the value of the Higgs-boson mass.

If both bottom jets accompanying the Higgs boson in the final state are tagged, one has to rely on the fully exclusive calculation for $g g \rightarrow b \bar{b}+h / H / A$. For the case of a single $b$ tag in the final state the corresponding calculation in the 5FS starts from the process $b g \rightarrow b+h / H / A$ with the final-state bottom quark carrying finite transverse momentum. The NLO QCD, electroweak, and NLO SUSY-QCD corrections to this process have been calculated [34].

State-of-the-art predictions as well as estimates of the corresponding parametric and theoretical uncertainties, based on the described strategies and quoted calculations, have been provided by the LHC Higgs Cross Section Working Group both for total [22] and differential [23,24] cross sections.

In this paper we are going to extend the previous 4FS calculations of $t \bar{t} / b \bar{b}+$ Higgs production cross sections at the LHC to include the full supersymmetric QCD (SUSY-QCD) corrections within the MSSM and separate the dominating universal part in terms of effective Yukawa couplings. The paper is organized as follows: in Sec. II we shall describe the calculation of the NLO supersymmetric QCD corrections. Numerical results for MSSM Higgsboson production at the LHC are presented in Sec. III. We conclude in Sec. IV.

\section{CALCULATIONAL DETAILS}

At leading order (LO) Higgs radiation off heavy quarks is described by the partonic processes $q \bar{q}, g g \rightarrow Q \bar{Q}+$ $h / H / A(Q=t, b)$, as depicted by the generic Feynman diagrams of Fig. 1(a).

The NLO corrections arise from virtual one-loop diagrams for the pure QCD part [see Fig. 1(b)], the genuine SUSY-QCD part [see Fig. 1(c)], the real corrections originating from gluon radiation [see Fig. 1(d)], and the crossed channel emerging from gluon-quark initial states [see Fig. 1(e)]. The details of our calculation of the pure QCD corrections have been described in Ref. [15] (see also Ref. [35] for the closely related calculation of chargedHiggs-boson production with top and bottom quarks). 
(a)

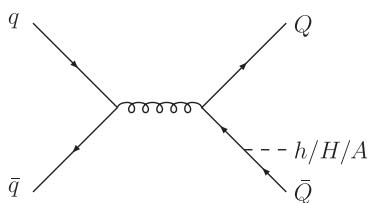

(b)

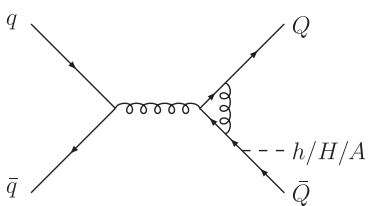

(c)

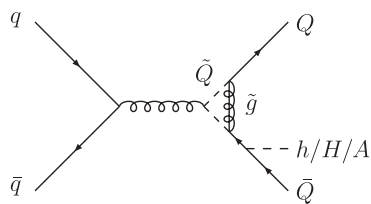

(d)

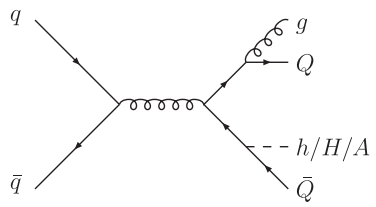

(e)

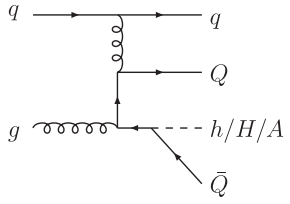

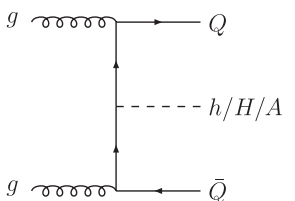
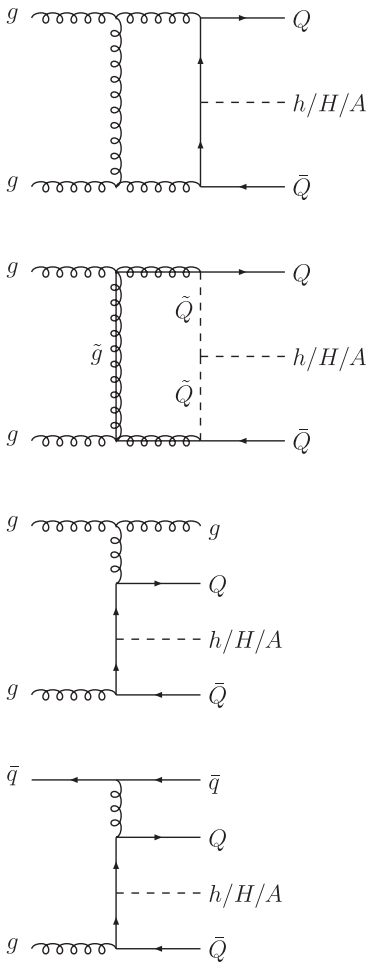

FIG. 1. A generic set of diagrams (a) for the Born level, (b) virtual gluon exchange, (c) virtual gluino and squark exchange, (d) gluon radiation, and (e) gluon-(anti)quark scattering in the subprocesses $q \bar{q}, g g \rightarrow Q \bar{Q}+h / H / A$, etc., where $Q=t, b$.

Building on our previous papers, we have extended the calculations to include the genuine SUSY-QCD corrections. A more detailed description of the corresponding computational details can be found in Ref. [17]. Two independent calculations of the SUSY-QCD corrections have been performed and found to be in mutual agreement.

The Feynman diagrams and amplitudes that contribute to the virtual SUSY-QCD corrections have been generated with FeynArts 3.2 [36]. The amplitudes have been processed further with two independent in-house Mathematica routines, which automatically create output in Fortran and $\mathrm{C}++$, respectively. The genuine virtual SUSY-QCD corrections are infrared finite, since all virtual particles inside the loop contributions are massive. The pentagon tensor integrals have been reduced directly to box integrals following Ref. [37]. This method does not introduce inverse Gram determinants in the reduction process, thereby avoiding numerical instabilities in regions where these determinants become small. Box and lower-point

integrals have been reduced to scalar integrals using the standard Passarino-Veltman technique [38]. Sufficient numerical stability is already achieved in this way, but further improvements could be achieved with the methods of Ref. [39] if needed in future evaluations. The scalar integrals, finally, have been calculated either analytically or using the results of Ref. [40]. The (IR-finite) scalar integrals have furthermore been checked with LoopTools/FF [41].

The renormalization of the strong coupling $\alpha_{\mathrm{s}}(\mu)$ and the factorization of initial-state collinear singularities are performed in the $\overline{\mathrm{MS}}$ scheme. The top quark and the SUSY particles are decoupled from the running of $\alpha_{\mathrm{s}}(\mu)$. In the 4FS calculation presented here for the $b \bar{b}+$ Higgs case, also the bottom quark is decoupled and the partonic cross section is calculated using a four-flavor $\alpha_{\mathrm{s}}$. While the top- and bottom-quark masses are defined on-shell, the $\overline{\mathrm{MS}}$ scheme is adopted for the renormalization of the bottom-Higgs Yukawa coupling, which is fixed in terms of the corresponding $\overline{\mathrm{MS}}$ renormalization of the bottom mass with decoupled SUSY contributions. In order to sum large logarithmic corrections $\propto \ln \left(m_{b} / \mu\right)$ we evaluate the Yukawa coupling with the running $b$-quark mass $\bar{m}_{b}(\mu)$ [42].

The SUSY loop corrections modify the tree-level relation between the bottom mass and its Yukawa coupling, which is enhanced at $\operatorname{large} \operatorname{tg} \beta$ [43]. These corrections can be summed to all orders by replacing the bottom Yukawa coupling coefficients of Table I by $[44,45]$

$$
\begin{aligned}
& \tilde{g}_{b}^{h}=\frac{g_{b}^{h}}{1+\Delta_{b}}\left(1-\frac{\Delta_{b}}{\operatorname{tg} \alpha \operatorname{tg} \beta}\right), \\
& \tilde{g}_{b}^{H}=\frac{g_{b}^{H}}{1+\Delta_{b}}\left(1+\Delta_{b} \frac{\operatorname{tg} \alpha}{\operatorname{tg} \beta}\right), \\
& \tilde{g}_{b}^{A}=\frac{g_{b}^{A}}{1+\Delta_{b}}\left(1-\frac{\Delta_{b}}{\operatorname{tg}^{2} \beta}\right),
\end{aligned}
$$

where

$$
\begin{aligned}
\Delta_{b} & =\frac{C_{F}}{2} \frac{\alpha_{\mathrm{s}}}{\pi} m_{\tilde{g}} \mu \operatorname{tg} \beta I\left(m_{\tilde{b}_{1}}^{2}, m_{\tilde{b}_{2}}^{2}, m_{\tilde{g}}^{2}\right), \\
I(a, b, c) & =\frac{a b \log (a / b)+b c \log (b / c)+c a \log (c / a)}{(a-b)(b-c)(a-c)}
\end{aligned}
$$

with $C_{F}=4 / 3$. Here, $\tilde{b}_{1,2}$ are the sbottom mass eigenstates, and $m_{\tilde{g}}$ denotes the gluino mass. The summation formalism can be extended [45] to include corrections proportional to the trilinear coupling $A_{b}$. However, for the MSSM scenarios under consideration in this work, these corrections turn out to be small, and the corresponding summation effects may safely be neglected. The scale of $\alpha_{\mathrm{s}}$ within the resummed bottom Yukawa couplings of Eq. (1) has been chosen as the average of the contributing SUSY masses $\mu=\left(m_{\tilde{b}_{1}}+m_{\tilde{b}_{2}}+m_{\tilde{g}}\right) / 3$, which is justified by the 
NNLO results for the $\Delta_{b}$ corrections [46]. Note that the resummed bottom Yukawa couplings have been defined by taking into account five active flavors. The strong coupling constant and the parton distribution functions (PDFs) involved in the explicit QCD and SUSY-QCD corrections to the production processes, on the other hand, are defined with four active flavors, analogous to the 4FS charged Higgs calculation discussed in some detail in Ref. [35].

If the LO cross section is expressed in terms of the bottom Yukawa couplings including the summation of the $\operatorname{tg} \beta$-enhanced corrections of Eq. (1), the corresponding NLO contributions have to be subtracted from the one-loop SUSY-QCD calculation to avoid double counting. This subtraction is equivalent to an additional finite renormalization of the bottom Yukawa coupling factors according to

$$
g_{b}^{\phi} \rightarrow \tilde{g}_{b}^{\phi}\left[1+\kappa_{\phi} \Delta_{b}\right]+\mathcal{O}\left(\alpha_{s}^{2}\right)
$$

with

$$
\begin{aligned}
& \kappa_{h}=1+\frac{1}{\operatorname{tg} \alpha \operatorname{tg} \beta}, \\
& \kappa_{H}=1-\frac{\operatorname{tg} \alpha}{\operatorname{tg} \beta}, \quad \text { and } \quad \kappa_{A}=1+\frac{1}{\operatorname{tg}^{2} \beta} .
\end{aligned}
$$

As we shall demonstrate in the numerical analysis presented in Sec. III, the SUSY-QCD radiative corrections are indeed sizable at large $\operatorname{tg} \beta$. After absorbing the $\operatorname{tg} \beta$ enhanced terms into effective Yukawa couplings, however, the remaining one-loop SUSY-QCD corrections are small, below the percent level, for scenarios with large SUSYparticle masses.

\section{NUMERICAL RESULTS}

To illustrate the impact of the NLO SUSY-QCD corrections we present numerical results for the LHC with $\mathrm{CM}$ energies of 7 and $14 \mathrm{TeV}$. Note that further results of our calculation, including those for $8 \mathrm{TeV}$ and a wider range of Higgs masses, will be available from the web pages of the LHC Higgs Cross Section Working Group [47]. The cross sections for $t \bar{t} \phi^{0}$ are presented in the 5FS, while for $b \bar{b} \phi^{0}$ production we have adopted the 4FS. We have used the corresponding five- and four-flavor MSTW2008 PDFs $[48,49]$ with their respective LO strong coupling normalized to $\alpha_{\mathrm{s}}\left(M_{Z}\right)=0.13939(0.13355)$ and NLO coupling normalized to $\alpha_{\mathrm{s}}\left(M_{Z}\right)=0.12018(0.11490)$ in the 5FS (4FS). The on-shell top mass has been adopted as $m_{t}=172.5 \mathrm{GeV}$ and the on-shell bottom mass as $m_{b}=4.75 \mathrm{GeV}$ corresponding to a $\overline{\mathrm{MS}}$ mass $\bar{m}_{b}\left(\bar{m}_{b}\right)=4.40 \mathrm{GeV}$. The pole masses enter the phase-space integration as well as the corresponding quark propagators of the virtual and real matrix elements. The top pole mass has been used in addition for the top Yukawa couplings, while the bottom Yukawa couplings have been evaluated in terms of the $\overline{\mathrm{MS}}$ mass as described above.
Our default choice for the renormalization and factorization scales is $\mu_{R}=\mu_{F}=m_{t}+M_{\phi} / 2$ for $t \bar{t} \phi^{0}$ production and $\mu_{R}=\mu_{F}=\left(2 m_{b}+M_{\phi}\right) / 4$ for the $b \bar{b} \phi^{0}$ case; see Refs. [15,25,35].

We have chosen the Snowmass point SPS5 for Higgs radiation off top quarks and SPS1b for the bottom quark case [50]. The MSSM parameters of these two benchmark scenarios are given by

$$
\begin{aligned}
\underline{\text { SPS } 5: \operatorname{tg} \beta} & =5, \quad \mu=639.8 \mathrm{GeV}, \\
A_{t} & =-1671.4 \mathrm{GeV}, \quad A_{b}=-905.6 \mathrm{GeV}, \\
m_{\tilde{g}} & =710.3 \mathrm{GeV}, \quad m_{\tilde{q}_{L}}=535.2 \mathrm{GeV}, \\
m_{\tilde{b}_{R}} & =620.5 \mathrm{GeV}, \quad m_{\tilde{t}_{R}}=360.5 \mathrm{GeV} . \\
\underline{\text { SPS1b: } \operatorname{tg} \beta} & =30, \quad \mu=495.6 \mathrm{GeV}, \\
A_{t} & =-729.3 \mathrm{GeV}, \quad A_{b}=-987.4 \mathrm{GeV}, \\
m_{\tilde{g}} & =916.1 \mathrm{GeV}, \quad m_{\tilde{q}_{L}}=762.5 \mathrm{GeV}, \\
m_{\tilde{b}_{R}} & =780.3 \mathrm{GeV}, \quad m_{\tilde{t}_{R}}=670.7 \mathrm{GeV} .
\end{aligned}
$$

The pseudoscalar Higgs mass is left as a free parameter in both scenarios in order to scan the Higgs mass ranges. The corresponding squark masses and couplings that enter our calculation have been calculated by using the tree-level relations. The Higgs masses and couplings have been determined from $\operatorname{tg} \beta$ and the pseudoscalar mass $M_{A}$ by taking into account higher-order corrections up to two loops in the effective potential approach [53] as included in the program HDECAY [54]. For the Higgs mass and coupling determination a five-flavor $\alpha_{\mathrm{s}}$ has been used normalized to $\alpha_{\mathrm{s}}\left(M_{Z}\right)=0.120$. The running bottom Yukawa couplings have been determined with $\alpha_{\mathrm{s}}\left(M_{Z}\right)=$ $0.120(0.139)$ at NLO (LO) with consistent NLO (LO) running.

The total cross sections for light and heavy scalar Higgs radiation off top quarks are shown at LO and NLO in Fig. 2(a) and the corresponding $K$ factors in Fig. 2(b). ${ }^{2}$ The contributions of the SUSY-QCD corrections to the $K$ factors, shown in the lower figure as dashed lines, amount to about $\pm 20 \%-30 \%$ and partially compensate the pure

\footnotetext{
${ }^{1}$ It should be noted that the Snowmass point SPS5 is excluded by the recent searches for squarks and gluinos by the ATLAS and CMS experiments [51] and the Snowmass point SPS1b is close to the recent exclusion contours. However, our results are only marginally affected qualitatively by slightly larger squark and gluino masses in general and thus serve as a guideline for scenarios beyond the present LHC reach; see e.g. Ref. [52].

${ }^{2}$ We have compared our calculation to the results for $t \bar{t} h$ production of Ref. [18]. Although we could not implement their scenario exactly in our calculation we have found reasonable agreement within a scenario very similar to theirs. The tiny SUSY-QCD corrections of Ref. [18] arise, since those authors work at the upper bound of the light scalar Higgs mass where the genuine SUSY-QCD corrections are small in our calculation, too; see Fig. 2(b).
} 

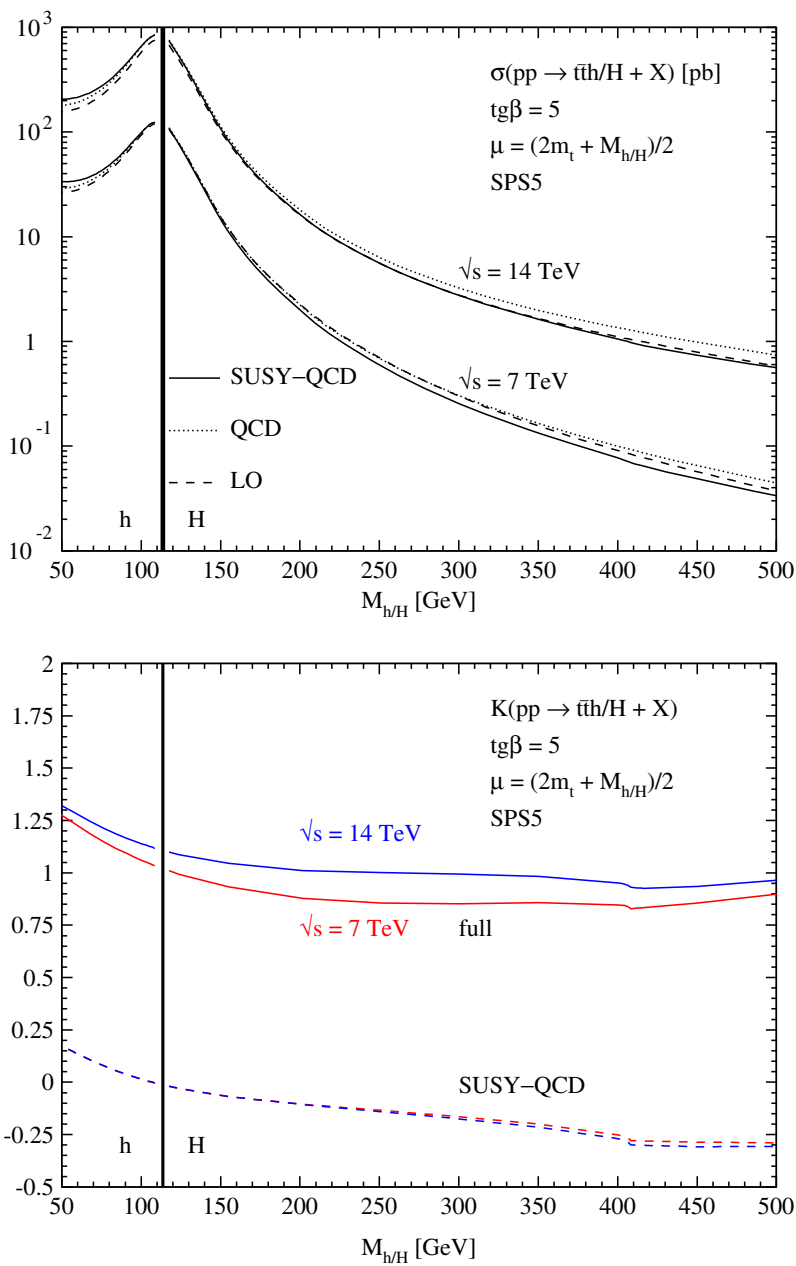

FIG. 2 (color online). SUSY-QCD corrected production cross sections of light and heavy scalar MSSM Higgs bosons in association with $t \bar{t}$ pairs for the Snowmass point SPS5 [50]. (a) Total cross sections. (b) $K$ factors.

QCD corrections for this MSSM scenario, thus leaving a moderate total correction to the cross sections as can be inferred from Fig. 2(b). The small kink at $M_{H} \sim 410 \mathrm{GeV}$ emerges from the virtual $\tilde{t}_{1} \overline{\tilde{t}}_{1}$ threshold ${ }^{3}$ inside the virtual loop contributions.

The total cross sections for pseudoscalar Higgs radiation off top quarks are displayed at LO and NLO in Fig. 3(a) and the corresponding $K$ factors in Fig. 3(b). Similar to the scalar case the SUSY-QCD corrections amount to about $-20 \%$ and compensate the pure QCD corrections for this MSSM scenario to a large extent, thus leaving a moderate total correction to the cross sections as shown in Fig. 3(b). The dependence of the $K$ factors on the LHC CM energy is weaker than in the scalar Higgs case [cf. Fig. 2(b)].

For Higgs-boson radiation off bottom quarks, we calculate the NLO cross section according to

\footnotetext{
${ }^{3}$ The light top squark mass amounts to $m_{\tilde{t}_{1}}=204.1 \mathrm{GeV}$ in the SPS5 scenario.
}
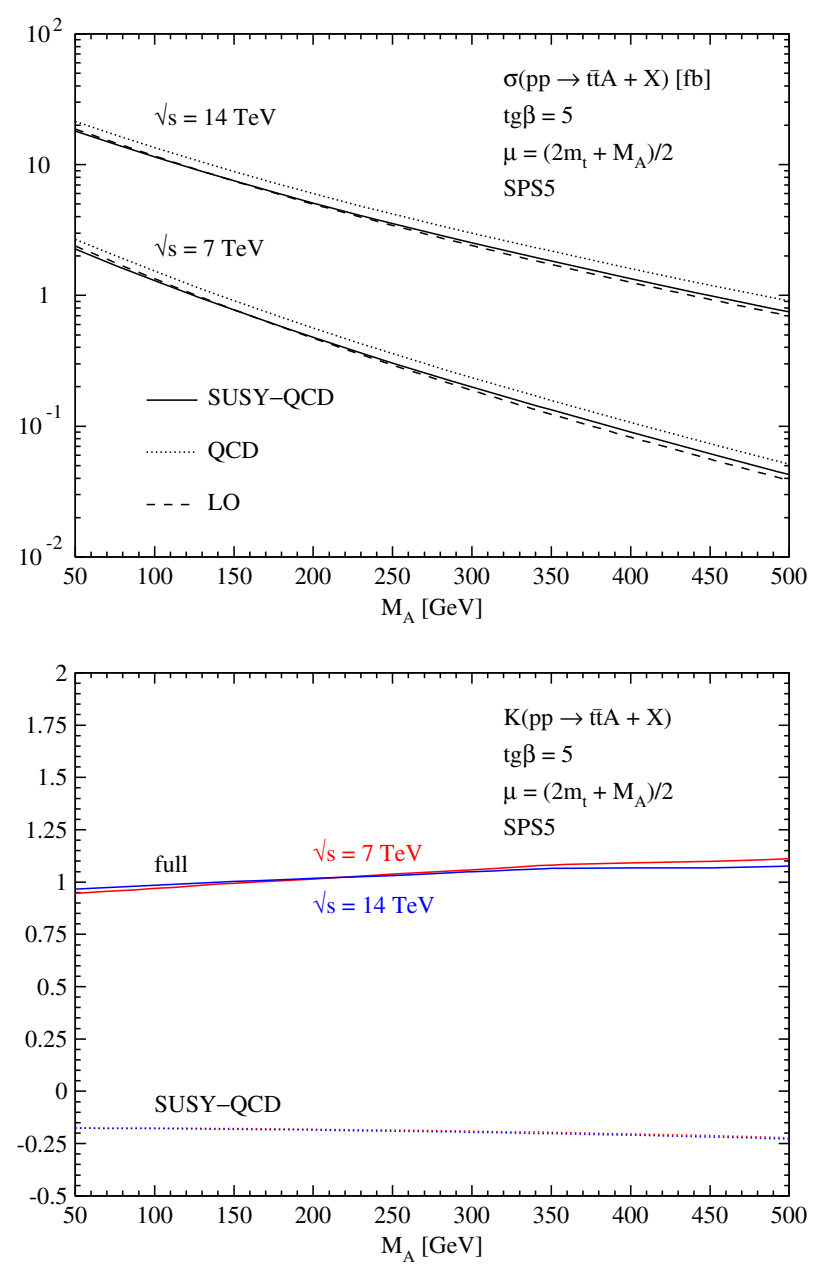

FIG. 3 (color online). SUSY-QCD corrected production cross sections of pseudoscalar MSSM Higgs bosons in association with $t \bar{t}$ pairs for the Snowmass point SPS5 [50]. (a) Total cross sections. (b) $K$ factors.

$$
\sigma_{\mathrm{NLO}}^{\phi}=\sigma_{0}^{\phi} \times\left(1+\delta_{\mathrm{SUSY}}^{\phi}\right) \times\left(1+\delta_{\mathrm{QCD}}^{\phi}+\delta_{\mathrm{SUSY}-\mathrm{rem}}^{\phi}\right)
$$

where $\sigma_{0}^{\phi}$ denotes the LO cross section evaluated with LO $\alpha_{\mathrm{s}}$ and PDFs, with the Yukawa coupling parametrized in terms of the running $b$-quark mass $\bar{m}_{b}(\mu)$, but without resummation of the $\operatorname{tg} \beta$-enhanced terms. The correction $\delta_{\text {SUSY }}^{\phi}$ comprises the $\operatorname{tg} \beta$-enhanced terms according to Eq. (1), including their resummation. The remainder of the genuine SUSY-QCD corrections is denoted by $\delta_{\text {SUSY-rem }}^{\phi}$.

The results for scalar Higgs-boson radiation off bottom quarks are shown in Figs. 4(a) (total cross section) and 4(b) ( $K$ factor). Here we identify the LO cross section with $\sigma_{0}^{\phi}$, which does not include the $\operatorname{tg} \beta$-enhanced resummation effects. The observed moderate NLO corrections in this MSSM scenario, thus, result from a compensation of the large QCD corrections by large SUSY-QCD corrections. The smallness of the SUSY-QCD remainder, however, 

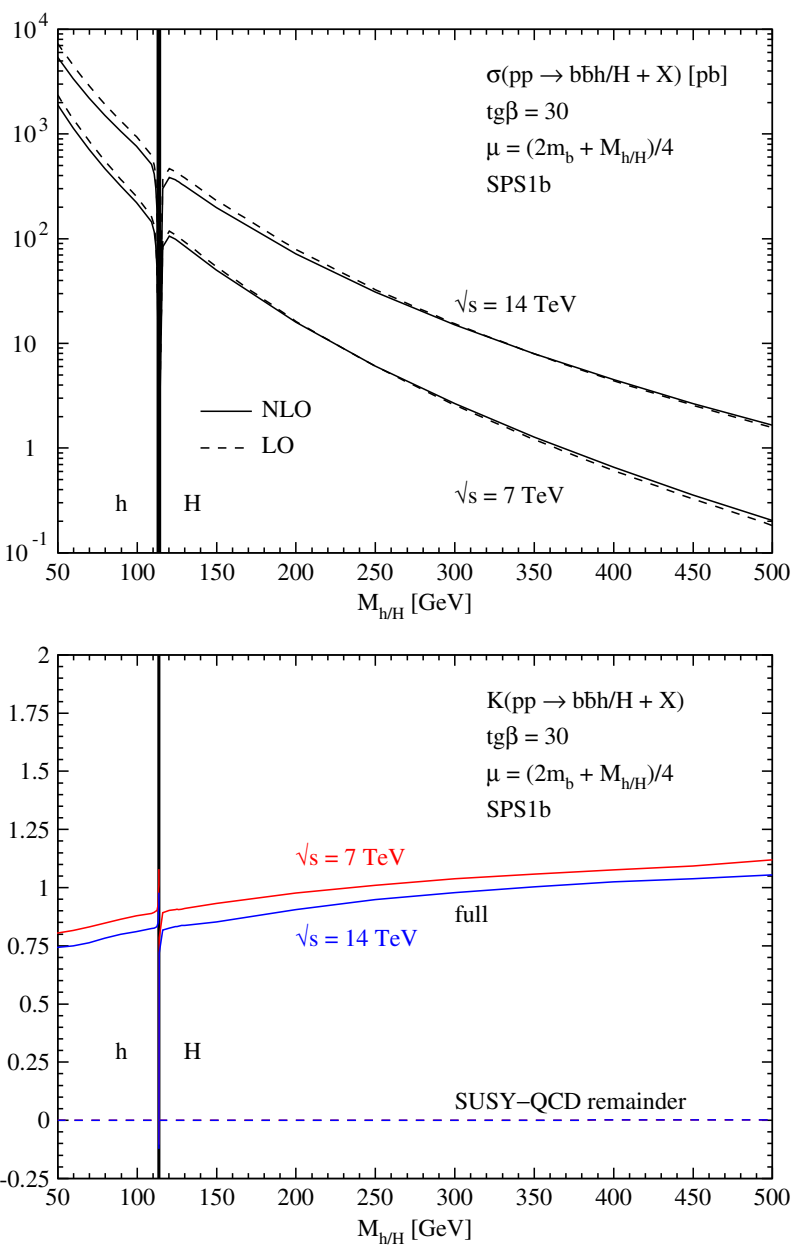

FIG. 4 (color online). SUSY-QCD corrected production cross sections of light and heavy scalar MSSM Higgs bosons in association with $b \bar{b}$ pairs for the Snowmass point SPS1b [50]. (a) Total cross sections. (b) $K$ factors.

shows that the full NLO SUSY-QCD corrections are approximated extremely well by the $\operatorname{tg} \beta$-enhanced terms.

Some numerical results for the SUSY-QCD corrections to the process $p p \rightarrow b \bar{b} h$ have been presented in Ref. [55]. However, only scatter plots are shown in [55], but no definite values for cross sections or SUSY-QCD corrections for a reference input. It is thus not possible to quantitatively compare our results to those of Ref. [55].

Pseudoscalar Higgs radiation off bottom quarks exhibits the same qualitative features as scalar Higgs production, as can be inferred from Fig. 5(a) for the total cross section and from Fig. 5(b) for the $K$ factor. The CM energy dependence of the $K$ factor for pseudoscalar Higgs production is similar to that of scalar Higgs production. The pseudoscalar-Higgsboson cross section at NLO coincides with the scalar cross sections for the same Higgs mass values within a few percent apart from the regions where the light (heavy) scalar Higgs boson is close to its upper (lower) mass bound.

In Table II we show the individual contributions to the NLO cross sections for heavy scalar and pseudoscalar
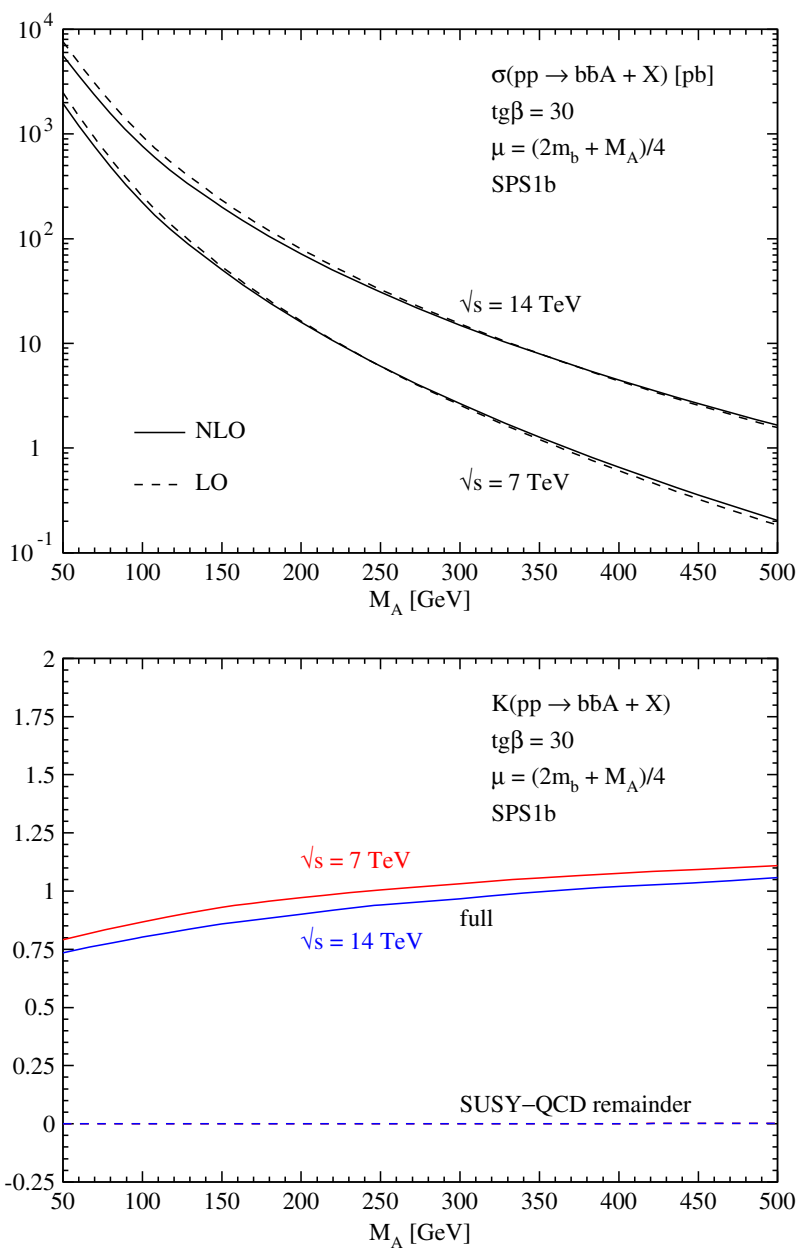

FIG. 5 (color online). SUSY-QCD corrected production cross sections of pseudoscalar MSSM Higgs bosons in association with $b \bar{b}$ pairs for the Snowmass point SPS1b [50]. (a) Total cross sections. (b) $K$ factors.

Higgs radiation off bottom quarks. The relative pure QCD corrections $\delta_{\mathrm{QCD}}^{\phi}(\phi=H, A)$, the $\operatorname{tg} \beta$-enhanced SUSY-QCD corrections $\delta_{\mathrm{SUSY}}^{\phi}$, and the remainders of the SUSY-QCD corrections $\delta_{\text {SUSY-rem }}^{\phi}$ are defined according to Eq. (5). As already observed in the corresponding figures, the sizable QCD corrections $\delta_{\mathrm{QCD}}^{\phi}$ are to a large extent compensated by the SUSY-QCD corrections $\delta_{\mathrm{SUSY}}^{\phi}$, thus leaving a small remainder $\delta_{\text {SUSY-rem }}^{\phi}$ of the SUSY-QCD corrections below the percent level for all Higgs masses in the SPS1b scenario.

In order to quantify the accuracy of the $\Delta_{b}$ approximation of Eq. (1) we display the effect of the $\operatorname{tg} \beta$ resummation and the corresponding remainders of the SUSY-QCD corrections for various values of $\operatorname{tg} \beta$ in Table III. For these numbers we have chosen the Snowmass point SPS1b, but set $M_{A}=200 \mathrm{GeV}$ and vary $\operatorname{tg} \beta$ between $3 \leq \operatorname{tg} \beta \leq 30$. The results demonstrate that the $\Delta_{b}$ approximation for the genuine SUSY-QCD corrections is accurate to better than 
TABLE II. Individual NLO corrections relative to the LO cross section for $p p \rightarrow b \bar{b} \phi+X(\phi=H, A)$, as defined in Eq. (1), are shown for the LHC at two CM energies (7 and $14 \mathrm{TeV}$ ) for the Snowmass point SPS1b [50].

\begin{tabular}{ccccccccc}
\hline \hline & $M_{A}$ & $M_{H}(\mathrm{GeV})$ & $\delta_{\mathrm{QCD}}^{A}$ & $\delta_{\text {SUSY }}^{A}$ & $\delta_{\text {SUSY rem }}^{A}$ & $\delta_{\text {QCD }}^{H}$ & $\delta_{\text {SUSY }}^{H}$ & $\delta_{\text {SUSY rem }}^{H}$ \\
\hline \multirow{4}{*}{$7 \mathrm{TeV}$} & 100 & 113.9 & 0.23 & -0.30 & $0.4 \times 10^{-4}$ & 0.27 & -0.38 & $0.3 \times 10^{-4}$ \\
& 200 & 200 & 0.38 & -0.30 & $2.9 \times 10^{-4}$ & 0.39 & -0.30 & $5.8 \times 10^{-4}$ \\
& 300 & 300 & 0.46 & -0.30 & $6.7 \times 10^{-4}$ & 0.47 & -0.30 & $9.3 \times 10^{-4}$ \\
& 400 & 400 & 0.53 & -0.30 & $1.3 \times 10^{-3}$ & 0.53 & -0.30 & $1.5 \times 10^{-3}$ \\
& 500 & 500 & 0.57 & -0.30 & $2.0 \times 10^{-3}$ & 0.59 & -0.30 & $2.2 \times 10^{-3}$ \\
$14 \mathrm{TeV}$ & 100 & 113.9 & 0.14 & -0.30 & $0.4 \times 10^{-4}$ & 0.17 & -0.38 & $0.5 \times 10^{-4}$ \\
& 200 & 200 & 0.28 & -0.30 & $2.7 \times 10^{-4}$ & 0.29 & -0.30 & $5.7 \times 10^{-4}$ \\
& 300 & 300 & 0.37 & -0.30 & $6.5 \times 10^{-4}$ & 0.39 & -0.30 & $9.3 \times 10^{-4}$ \\
& 400 & 400 & 0.45 & -0.30 & $1.2 \times 10^{-3}$ & 0.45 & -0.30 & $1.5 \times 10^{-3}$ \\
\hline \hline
\end{tabular}

TABLE III. The $\operatorname{tg} \beta$ dependence of the individual genuine SUSY-QCD corrections relative to the LO cross section for $p p \rightarrow b \bar{b} \phi+X$ $(\phi=H, A)$ at the LHC for two CM energies ( 7 and $14 \mathrm{TeV}$ ). The Snowmass point SPS1b [50] is adopted with $M_{A}$ and tg $\beta$ left as free parameters. The pseudoscalar Higgs mass is fixed at $200 \mathrm{GeV}$.

\begin{tabular}{rrrccccc}
\hline \hline & $\operatorname{tg} \beta$ & $M_{A}$ & $M_{H}(\mathrm{GeV})$ & $\delta_{\text {SUSY }}^{A}$ & $\delta_{\text {SUSY rem }}^{A}$ & $\delta_{\text {SUSY }}^{H}$ & $\delta_{\text {SUSY rem }}^{H}$ \\
\hline \multirow{4}{*}{$7 \mathrm{TeV}$} & 3 & 200 & 209.7 & -0.04 & $2.1 \times 10^{-4}$ & -0.04 & $5.7 \times 10^{-4}$ \\
& 5 & 200 & 204.0 & -0.06 & $2.4 \times 10^{-4}$ & -0.06 & $5.3 \times 10^{-4}$ \\
& 7 & 200 & 202.1 & -0.08 & $2.5 \times 10^{-4}$ & -0.09 & $3.9 \times 10^{-4}$ \\
& 10 & 200 & 200.9 & -0.12 & $2.5 \times 10^{-4}$ & -0.12 & $3.8 \times 10^{-4}$ \\
& 20 & 200 & 200.1 & -0.21 & $2.6 \times 10^{-4}$ & -0.21 & $4.4 \times 10^{-4}$ \\
& 30 & 200 & 200.0 & -0.30 & $2.9 \times 10^{-4}$ & -0.30 & $5.8 \times 10^{-4}$ \\
& 3 & 200 & 209.7 & -0.04 & $2.0 \times 10^{-4}$ & -0.04 & $7.2 \times 10^{-4}$ \\
$14 \mathrm{TeV}$ & 5 & 200 & 204.0 & -0.06 & $2.2 \times 10^{-4}$ & -0.06 & $5.0 \times 10^{-4}$ \\
& 7 & 200 & 202.1 & -0.08 & $2.4 \times 10^{-4}$ & -0.09 & $4.4 \times 10^{-4}$ \\
& 10 & 200 & 200.9 & -0.12 & $2.5 \times 10^{-4}$ & -0.12 & $4.1 \times 10^{-4}$ \\
& 20 & 200 & 200.1 & -0.21 & $2.7 \times 10^{-4}$ & -0.21 \\
\hline \hline
\end{tabular}

the percent level for all values of $\operatorname{tg} \beta$, at least in MSSM scenarios with heavy SUSY particles. Similar results have been obtained for other Higgs masses within the SPS1b scenario.

\section{CONCLUSIONS}

We have presented the next-to-leading order supersymmetric QCD corrections to neutral MSSM Higgs-boson production at the LHC through the parton processes $q \bar{q}, g g \rightarrow Q \bar{Q}+h / H / A \quad(Q=t, b)$. For neutral Higgs radiation off top quarks we find moderate SUSY-QCD corrections, similar in magnitude to the pure QCD effects. Depending on the MSSM scenario, the genuine SUSYQCD corrections can be positive or negative. On the other hand, the genuine SUSY-QCD corrections to neutral Higgs radiation off bottom quarks turn out to be large for large values of $\operatorname{tg} \beta$, due to $\operatorname{large} \operatorname{tg} \beta$-enhanced contributions.
They can thus significantly change the large pure QCD corrections. The dominant part of these genuine SUSYQCD corrections can be absorbed in effective bottom Yukawa couplings which resum the $\operatorname{tg} \beta$-enhanced corrections. Although not explicitly shown in this work the same conclusions hold for the differential cross sections. The NLO results obtained in this work will be important for a reliable extraction of the SUSY parameters from future LHC data if the new particle with mass $\approx 125 \mathrm{GeV}$ turns out to be a supersymmetric Higgs boson.

\section{ACKNOWLEDGMENTS}

This work has been supported by the DFG SFB/TR9 "Computational Particle Physics." The research of S. D. and M. S. is supported in part by the European Commission through the "HiggsTools" Initial Training Network PITNGA-2012-316704. 
[1] P. W. Higgs, Phys. Lett. 12, 132 (1964); Phys. Rev. Lett. 13, 508 (1964); Phys. Rev. 145, 1156 (1966); F. Englert and R. Brout, Phys. Rev. Lett. 13, 321 (1964); G. S. Guralnik, C. R. Hagen, and T. W. Kibble, Phys. Rev. Lett. 13, 585 (1964).

[2] ATLAS Collaboration, Phys. Lett. B 716, 1 (2012); S. Chatrchyan et al. (CMS Collaboration), Phys. Lett. B 716, 30 (2012).

[3] P. Fayet, Nucl. Phys. B90, 104 (1975); Phys. Lett. 64B, 159 (1976); 69B, 489 (1977); S. Dimopoulos and H. Georgi, Nucl. Phys. B193, 150 (1981); N. Sakai, Z. Phys. C 11, 153 (1981); K. Inoue, A. Kakuto, H. Komatsu, and S. Takeshita, Prog. Theor. Phys. 67, 1889 (1982); 70, 330 (1983); 71, 413 (1984).

[4] See e.g. G. Degrassi, S. Heinemeyer, W. Hollik, P. Slavich, and G. Weiglein, Eur. Phys. J. C 28, 133 (2003).

[5] S. P. Martin, Phys. Rev. D 75, 055005 (2007); R. V. Harlander, P. Kant, L. Mihaila, and M. Steinhauser, Phys. Rev. Lett. 100, 191602 (2008); 101, 039901(E) (2008); P. Kant, R. V. Harlander, L. Mihaila, and M. Steinhauser, J. High Energy Phys. 08 (2010) 104.

[6] R. Barbieri, M. Frigeni, and F. Caravaglios, Phys. Lett. B 258, 167 (1991); Y. Okada, M. Yamaguchi, and T. Yanagida, Phys. Lett. B 262, 54 (1991); D. M. Pierce, A. Papadopoulos, and S. Johnson, Phys. Rev. Lett. 68, 3678 (1992); K. Sasaki, M. S. Carena, and C. E. M. Wagner, Nucl. Phys. B381, 66 (1992); J. Kodaira, Y. Yasui, and K. Sasaki, Phys. Rev. D 50, 7035 (1994); J. R. Espinosa and M. Quiros, Phys. Lett. B 266, 389 (1991); H. E. Haber and R. Hempfling, Phys. Rev. D 48, 4280 (1993).

[7] T. Hahn, S. Heinemeyer, W. Hollik, H. Rzehak, and G. Weiglein, Phys. Rev. Lett. 112, 141801 (2014).

[8] See e.g. M. Spira and P. M. Zerwas, Lect. Notes Phys. 512, 161 (1998); M. Gomez-Bock, M. Mondragon, M. Mühlleitner, R. Noriega-Papaqui, I. Pedraza, M. Spira, and P. M. Zerwas, J. Phys. Conf. Ser. 18, 74 (2005).

[9] S. Schael et al. (ALEPH, DELPHI, L3, and OPAL Collaborations), Eur. Phys. J. C 47, 547 (2006).

[10] T. Aaltonen et al. (CDF and D0 Collaborations), Phys. Rev. D 86, 091101 (2012).

[11] ATLAS Collaboration, Technical Design Report No. CERN-LHCC 99-14, 1999; G. L. Bayatian et al. (CMS Collaboration), J. Phys. G 34, 995 (2007).

[12] ATLAS Collaboration, J. High Energy Phys. 02 (2013) 095; S. Chatrchyan et al. (CMS Collaboration), Phys. Lett. B 713, 68 (2012); 722, 207 (2013).

[13] M. Spira, Fortschr. Phys. 46, 203 (1998); A. Djouadi, Phys. Rep. 459, 1 (2008).

[14] R. Raitio and W. W. Wada, Phys. Rev. D 19, 941 (1979); J. N. Ng and P. Zakarauskas, Phys. Rev. D 29, 876 (1984); Z. Kunszt, Nucl. Phys. B247, 339 (1984); J. F. Gunion, Phys. Lett. B 261, 510 (1991); W. J. Marciano and F. E. Paige, Phys. Rev. Lett. 66, 2433 (1991).

[15] W. Beenakker, S. Dittmaier, M. Krämer, B. Plümper, M. Spira, and P. Zerwas, Phys. Rev. Lett. 87, 201805 (2001); Nucl. Phys. B653, 151 (2003).

[16] L. Reina and S. Dawson, Phys. Rev. Lett. 87, 201804 (2001); S. Dawson, L. H. Orr, L. Reina, and D. Wackeroth, Phys. Rev. D 67, 071503 (2003).

[17] P. Häfliger, Ph.D. thesis, Eidgenössische Technische Hochschule, Zürich, DISS-ETH-16970, 2006; M. Walser, Ph.D. thesis, ETH, Zürich, DISS-ETH-17592, 2008.
[18] W. Peng, M. Wen-Gan, H. Hong-Sheng, Z. Ren-You, H. Liang, and J. Yi, Phys. Lett. B 618, 209 (2005).

[19] W. Hollik and M. Rauch, AIP Conf. Proc. 903, 117 (2007).

[20] R. Frederix, S. Frixione, V. Hirschi, F. Maltoni, R. Pittau, and P. Torrielli, Phys. Lett. B 701, 427 (2011); M. V. Garzelli, A. Kardos, C. G. Papadopoulos, and Z. Trocsanyi, Europhys. Lett. 96, 11001 (2011).

[21] A. Bredenstein, A. Denner, S. Dittmaier, and S. Pozzorini, Phys. Rev. Lett. 103, 012002 (2009); J. High Energy Phys. 03 (2010) 021; G. Bevilacqua, M. Czakon, C. G. Papadopoulos, R. Pittau, and M. Worek, J. High Energy Phys. 09 (2009) 109; Phys. Rev. Lett. 104, 162002 (2010); G. Bevilacqua, M. Czakon, C. G. Papadopoulos, and M. WorekPhys. Rev. D 84, 114017 (2011); S. Hoeche et al., arXiv:1402.6293; G. Bevilacqua and M. Worek, arXiv:1403.2046 [J. High Energy Phys. (to be published)].

[22] S. Dittmaier, C. Mariotti, G. Passarino, R. Tanaka et al. (LHC Higgs Cross Section Working Group Collaboration), arXiv:1101.0593.

[23] S. Dittmaier, C. Mariotti, G. Passarino, R. Tanaka et al. (LHC Higgs Cross Section Working Group Collaboration), arXiv:1201.3084.

[24] S. Heinemeyer, C. Mariotti, G. Passarino, R. Tanaka et al. (LHC Higgs Cross Section Working Group Collaboration), arXiv:1307.1347.

[25] S. Dittmaier, M. Krämer, and M. Spira, Phys. Rev. D 70, 074010 (2004); S. Dawson, C. Jackson, L. Reina, and D. Wackeroth, Phys. Rev. D 69, 074027 (2004).

[26] R. M. Barnett, H. E. Haber, and D. E. Soper, Nucl. Phys. B306, 697 (1988).

[27] V. N. Gribov and L. N. Lipatov, Yad. Fiz. 15, 781 (1972) [Sov. J. Nucl. Phys. 15, 438 (1972)]; G. Altarelli and G. Parisi, Nucl. Phys. B126, 298 (1977); Y. L. Dokshitzer, Zh. Eksp. Teor. Fiz. 73, 1216 (1977) [Sov. Phys. JETP 46, 641 (1977)].

[28] D. A. Dicus and S. Willenbrock, Phys. Rev. D 39, 751 (1989).

[29] D. Dicus, T. Stelzer, Z. Sullivan, and S. Willenbrock, Phys. Rev. D 59, 094016 (1999); C. Balazs, H.-J. He, and C. Yuan, Phys. Rev. D 60, 114001 (1999).

[30] R. V. Harlander and W. B. Kilgore, Phys. Rev. D 68, 013001 (2003).

[31] S. Dittmaier, M. Krämer, A. Mück, and T. Schlüter, J. High Energy Phys. 03 (2007) 114.

[32] J. M. Campbell et al., arXiv:hep-ph/0405302; S. Dawson, C. B. Jackson, L. Reina, and D. Wackeroth, Mod. Phys. Lett. A 21, 89 (2006).

[33] R. Harlander, M. Krämer, and M. Schumacher, arXiv:1112.3478.

[34] J. M. Campbell, R. Ellis, F. Maltoni, and S. Willenbrock, Phys. Rev. D 67, 095002 (2003); S. Dawson, C. Jackson, L. Reina, and D. Wackeroth, Phys. Rev. Lett. 94, 031802 (2005); M. Beccaria, G. Dovier, G. Macorini, E. Mirabella, L. Panizzi, F. M. Renard, and C. Verzegnassi, Phys. Rev. D 82, 093018 (2010); S. Dawson and C. Jackson, Phys. Rev. D 77, 015019 (2008).

[35] S. Dittmaier, M. Krämer, M. Spira, and M. Walser, Phys. Rev. D 83, 055005 (2011); S. Dittmaier, M. Krämer, M. Spira, and M. WalserProc. Sci., RADCOR 2009 (2010) 046. 
[36] J. Küblbeck, M. Böhm, and A. Denner, Comput. Phys. Commun. 60, 165 (1990); H. Eck and J. Küblbeck, Guide to FeynArts 1.0 (University of Würzburg, Würzburg, 1992); T. Hahn, Comput. Phys. Commun. 140, 418 (2001).

[37] A. Denner and S. Dittmaier, Nucl. Phys. B658, 175 (2003).

[38] G. Passarino and M. Veltman, Nucl. Phys. B160, 151 (1979).

[39] A. Denner and S. Dittmaier, Nucl. Phys. B734, 62 (2006).

[40] G. 't Hooft and M. Veltman, Nucl. Phys. B153, 365 (1979); W. Beenakker and A. Denner, Nucl. Phys. B338, 349 (1990); A. Denner, U. Nierste, and R. Scharf, Nucl. Phys. B367, 637 (1991).

[41] T. Hahn and M. Perez-Victoria, Comput. Phys. Commun. 118, 153 (1999); G. J. van Oldenborgh, Comput. Phys. Commun. 66, 1 (1991).

[42] E. Braaten and J. P. Leveille, Phys. Rev. D 22, 715 (1980); M. Drees and K. I. Hikasa, Phys. Lett. B 240, 455 (1990); 262, 497 (1991); N. Sakai, Phys. Rev. D 22, 2220 (1980); T. Inami and T. Kubota, Nucl. Phys. B179, 171 (1981); S. G. Gorishnii, A. L. Kataev, and S. A. Larin, Yad. Fiz. 40, 517 (1984) [Sov. J. Nucl. Phys. 40, 329 (1984)].

[43] L. J. Hall, R. Rattazzi, and U. Sarid, Phys. Rev. D 50, 7048 (1994); R. Hempfling, Phys. Rev. D 49, 6168 (1994); M. Carena, M. Olechowski, S. Pokorski, and C. E. M. Wagner, Nucl. Phys. B426, 269 (1994); D. M. Pierce, J. A. Bagger, K. T. Matchev, and R. J. Zhang, Nucl. Phys. B491, 3 (1997).

[44] M. Carena, D. Garcia, U. Nierste, and C. E. M. Wagner, Nucl. Phys. B577, 88 (2000).

[45] J. Guasch, P. Häfliger, and M. Spira, Phys. Rev. D 68, 115001 (2003).
[46] D. Noth and M. Spira, Phys. Rev. Lett. 101, 181801 (2008); J. High Energy Phys. 06 (2011) 084; L. Mihaila and C. Reisser, J. High Energy Phys. 08 (2010) 021.

[47] https://twiki.cern.ch/twiki/bin/view/LHCPhysics/ CrossSections.

[48] A. D. Martin, W. J. Stirling, R. S. Thorne, and G. Watt, Eur. Phys. J. C 63, 189 (2009).

[49] A. D. Martin, W. J. Stirling, R. S. Thorne, and G. Watt, Eur. Phys. J. C 70, 51 (2010).

[50] B. C. Allanach et al., in Proceedings of the APS/DPF/DPB Summer Study on the Future of Particle Physics, Snowmass, 2001, edited by N. Graf [Eur. Phys. J. C 25, 113 (2002)]; eConf C010630, P125 (2001).

[51] ATLAS Collaboration, J. High Energy Phys. 10 (2013) 130; S. Chatrchyan et al. (CMS Collaboration), J. High Energy Phys. 06 (2014) 055.

[52] S. S. AbdusSalam, B. C. Allanach, H. K. Dreiner, J. Ellis, U. Ellwanger, J. Gunion, S. Heinemeyer, M. Krämer et al., Eur. Phys. J. C 71, 1835 (2011).

[53] M. S. Carena, J. R. Espinosa, M. Quiros, and C. E. M. Wagner, Phys. Lett. B 355, 209 (1995); M. Carena, H. E. Haber, S. Heinemeyer, W. Hollik, C. E. Wagner, G. Weiglein, Nucl. Phys. B580, 29 (2000); H. E. Haber, R. Hempfling, and A. H. Hoang, Z. Phys. C 75, 539 (1997).

[54] A. Djouadi, J. Kalinowski, and M. Spira, Comput. Phys. Commun. 108, 56 (1998); A. Djouadi, J. Kalinowski, M. Mühlleitner, and M. Spira, arXiv:1003.1643.

[55] N. Liu, L. Wu, P. W. Wu, and J. M. Yang, J. High Energy Phys. 01 (2013) 161. 